\title{
Reduced basis method for frequency sweeps with integral equations using locally adaptive kernel approximation
}

\author{
Philip Edel $^{\dagger *}$, Yvon Maday ${ }^{\star}$ and François-Xavier Roux ${ }^{\dagger}$ \\ $\dagger$ The French Aerospace Lab - ONERA \\ Chemin de la Hunière, 91120 Palaiseau, France \\ e-mail: philip.edel@onera.fr \\ * Sorbonne Université, CNRS, Université de Paris, Laboratoire Jacques Louis-Lions (LJLL), \\ Institut Universitaire de France \\ 75005 Paris, France
}

\begin{abstract}
Many electromagnetic and acoustic applications require the ability to explore all solutions in a given frequency window. When the problem is large scale, strategies based on computing a large number of solutions from successive solver calls usually lead to prohibitive computational costs. This is especially the case when the solver relies on integral equations discretized using the boundary element method (BEM), as this amounts to solving numerous complex, unsymmetrical and fully populated linear systems.
\end{abstract}

The reduced basis method (RBM) is an efficient approach to rapidly and accurately approximate any solution within a given frequency window $[1,2]$. In the context of frequency sweeps with the BEM, the success of the RBM essentially depends on the ability to decouple the frequency from the kernel of the underlying integral equation.

In this talk, we present a novel approach based on the ideas of local adaptivity [3]. The main benefit is that the overall number of operators to be assembled throughout the RBM (or to be computed using adequate low-rank approximation methods) is significantly reduced, compared to previous approaches $[1,4]$. The proposed methodology is illustrated with the double-layer operator on an academic problem.

\section{REFERENCES}

[1] M Fares, Jan S Hesthaven, Yvon Maday, and Benjamin Stamm. The reduced basis method for the electric field integral equation. Journal of Computational Physics, 230(14):5532-5555, 2011.

[2] Jan S Hesthaven, Benjamin Stamm, and SHUN Zhang. Certified reduced basis method for the electric field integral equation. SIAM Journal on Scientific Com- puting, 34(3):A1777-A1799, 2012.

[3] Yvon Maday and Benjamin Stamm. Locally adaptive greedy approximations for anisotropic parameter reduced basis spaces. SIAM Journal on Scientific Computing, 35(6):A2417-A2441, 2013.

[4] Jens L Eftang and Benjamin Stamm. Parameter multi-domain 'hp' empirical interpo- lation. International Journal for Numerical Methods in Engineering, 90(4):412-428, 2012. 